\title{
Nomen - omen? Titeländerungen in der kirchlichen Presse ${ }^{1}$ von Živan Bezić
}

Von alters her weiß und sagt man "Tempora mutantur et nos in illis“. Nur, daß das Tempo dieser Veränderungen heute viel schneller geworden ist. Die Eile und die Veränderungen auf allen Gebieten des menschlichen Lebens sind die auffallendsten Merkmale unserer Zeit. Sturm und Unruhe sind in die alte, geruhsame Festung Kirche eingedrungen. An allen Seiten dieser Festung finden Restaurationsarbeiten und Veränderungen statt, Türen und Fenster werden breiter gemacht, und die Lüftung wird verbessert. Altertümliche Farben werden mit neuen Farben übermalt, der Burggraben wird verschüttet und Brücken werden herabgelassen. Die Kirche wird heute zu einem offenen Schloß ohne Wassergräben und Umzäunung, sie wird zur Keuzung aller Verkehrsstraßen.

Neben den neuen Massenmedien, deren sich die Kirche bedient, kommt es auch bei den alten Massenmedien zu sichtbaren Veränderungen. So hat z. B. die Kirchenpresse ein ganz neues Gesicht bekommen. Auf diese Erscheinung möchten wir eingehen. Unter den zahlreichen Neuerungen, die man in der kirchlichen Publizistik antrifft, ist folgende Erscheinung besonders auffallend: Änderungen der Titel kirchlicher Publikationen. Statt der früheren, komprimittierenden Titel werden neue, anziehendere Benennungen gewählt. Zahl und Anteilwerte der Titeländerungen habe ich nicht exakt festgestellt. Die Beobachtungen beruhen auf Notizen kirchlicher Zeitschriften. ${ }^{2}$ Diese Erscheinung würde eine eingehendere Studie verdienen. Die Beispiele, die ich anführen werde, sind nur ein kleiner Teil der an den Titeln der kirchlichen Presse vorgenommenen Änderungen.

Das Phänomen hat - wie jede gesellschaftliche Erscheinung - einen tieferen Grund. Wenn für ein neuzugründendes Blatt ein Titel gesucht wird, müssen sich die Redakteure ziemliche Mühe geben, um für dieses Blatt einen Namen zu finden, der so getreu wie möglich ihre Ideen und Aufgaben ausdrücken wird. Verspüren sie aber das Bedürfnis, seinen Titel zu verändern, so bedeutet das, daß er nicht mehr diesem Zweck entspricht - oder, daß sich die Ideen der Redakteure verändert haben. Um so mehr kommt dem Bedeutung zu, als das Umtaufen keine christliche Gepflogenheit ist. Wir werden zuerst die Tatsachen anführen und dann ihre Ursachen zu ergründen versuchen.

\section{Nomen}

Mit den Titeländerungen begannen als erste die Niederländer. Die älteste holländische katholische Zeitschrift war "Tijdschrift ter voortplanting der godsdienstige vereering van het $H$. Hart van Jezus on van den $H$. Joseph". Sie erschien erstmals 1869 und beschäftigte sich mit Fragen des geistlichen Lebens. Schon 1873 bekam sie den neuen Titel „Maandrozen ter eere van het $H$. Hart van Jezus en ter verbreiding van het

Živan Bezić ist Theologieprofessor in Split/Jugoslawien. Seinen Beitrag veröffentlichen wir als Zeugnis der Aufmerksamkeit, mit der trotz schwieriger Quellenlage die Entwicklung der internationalen katholischen Pressepublizistik von einem sozialistischen Land aus beobachtet wird. 
Apostolaat des Gebeds“. 1916 wechselte sie zu „De Heraut van het H. Hart van Jezus“, ab 1966 hieß sie nur noch „De Heraut“ (= Der Herold).

Ein ähnliches Schicksal erlebte auch die dominikanische Wochenzeitung „De Bazuin“. Sie begann 1911 als „R. K. Advertentieblad De Reklame-Bazuin“, nannte sich dann ${ }_{n}$ R. K. Weekblad De Bazuin“ (1913), „R. K. Weekblad De Bazuin, Algemeen Orgaan voor Katholieken“ (1915), dann „De Bazuin. Allgemeen Weekblad voor Katholieken“ (1916), dann „De Bazuin. Weekblad voor Katholieken en zoekenden naar de Waarheid“ (1922), „De Bazuin. Populair Godsdienstwetenschappelijk en Apologetisch Weekblad voor Nederland en Koloniën“ (1929), „De Bazuin. Populair Apologetisch Weekblad" (1940) (von Oktober 1941 bis zum 3. Januar 1948 nicht erschienen), dann "De Bazuin. Populair Godsdienstig Weekblad voor Katholieken“ (1948, Nr. 1-7), dann „De Bazuin. Godsdienstig Weekblad voor Katholieken, Doopleerlingen en Zoekenden naar de Waarheid“ (1948, Nr. 8 ff.) und "De Bazuin. Weekblad voor Geloofsverkondiging“ (1948 ab. 1. November), dann "De Bazuin. Tussen Kerk en Wereld“ (1959) und schließlich nur "De Bazuin" (= Trompete, ab 1961).

Das von den Jesuiten gegründete niederländische Blatt hat den Titel nicht verändert, es hat ihn nur durch ein einziges, aber bedeutendes Wort erweitert. Früher nannte es sich nämlich „De Linie“, aber ab 1963 trägt es den Titel „De nieuwe Linie“ und wird nicht mehr von den Jesuiten geleitet.

Die Zeitschrift der niederländischen Karmeliten hatte 1947 - bei Erscheinungsbeginn - einen einfachen und klaren Titel: "Carmel. Tijdschrift voor geestelijk leven." Aber 1969 wurde sie zu „Speling. Tijdschrift voor bezinning “ umgetauft, was soviel wie Raum (schaffen), Spielraum und das Spielerische heißt. Mit der Titeländerung hat sich auch vollkommen der Inhalt gewandelt. Bis 1965 gaben in Holland 7 verschiedene Ordensgemeinschaften ihre eigenen Monatszeitschriften heraus, von denen die Mehrzahl dahinsiechte. Sie beschlossen ihre eigenen Zeitschriften einzustellen und eine gemeinsam mit größerer Auflage und dem Titel „Kruispunt“ zu gründen, was soviel wie Kreuzung heißt.

$\mathrm{Da}$ es in Deutschland viel mehr katholische Periodika gibt, kam es dort auch zu zahlreicheren Titeländerungen. Als eine der ersten hat ihren Titel die Monatszeitschrift der Jesuiten „Stimmen aus Maria Laach“ (1871 gegr.) geändert. Schon 1914 hieß sie "Stimmen der Zeit" (in Freiburg i. B. herausgegeben). Die Herder-Wochenzeitung "Der Christliche Sonntag“ änderte vor 8 Jahren ihren Titel zu "Christ in der Gegenwart". Die Zeitschrift einer katholischen Laiengruppe in Deutschland nannte sich bis 1961 „Werkhefte katholischer Laien“, hat aber jetzt fast jede Verbindung mit der Kirche abgebrochen und nennt sich nunmehr "Werkhefte“.

Die österreichische Pastoral-Zeitschrift „Der Seelsorger“ vereinigte sich 1970 mit der deutsch-schweizerischen „Diakonia“ unter Hinzufügung des Titels „Der Seelsorger“. Interessant ist, daß sich die „Diakonia“ früher "Anima“ betitelte; 1966 gab sie aber diesen Namen auf. 1972 fiel von der gemeinsamen Monatszeitschrift der zweite Teil des Titels, „Der Seelsorger", weg. So hat die „Diakonia“ Seele wie Seelsorger geschluckt.

Eine informative Zeitschrift des Verlagshauses Herder wurde sofort nach dem Zweiten Weltkrieg in zwei Ausgaben gedruckt. Die deutsche Ausgabe trug den Titel "Herder Korrespondenz" mit dem Untertitel "Orbis Catholicus“. Bei der österreichischen Ausgabe war es genau umgekehrt. Ab 1968 sind beide Ausgaben ver- 
einigt. Der Untertitel „Orbis Catholicus“ entfiel, der neue Untertitel heißt „Monatshefte für Gesellschaft und Religion“.

Die bekannte Publikation der deutschen kulturellen Laienbewegung, „Hochland“, hat 1972 sowohl die Redaktion als auch den Titel geändert. Jetzt nennt sie sich „Neues Hochland". Die Revue "Theologie und Philosophie", herausgegeben von der jesuitischen theologischen Hochschule in St. Georgen (Frankfurt), hieß früher "Scholastik“.

1968 hatte der deutsche Episkopat die katholische Wochenzeitung „Publik“ gegründet. Der Versuch ist aus mehreren Gründen, vor allem finanziellen, nicht gelungen. Als der Episkopat diese Wochenzeitung nicht mehr unterstützen wollte, kam es auf dem "linken Flügel" zum großen Sturm, und eine Gruppe von Theologie-Studenten gründete mit Hilfe früherer "Publik“-Redakteure eine neue Zeitschrift, „Publik-Forum". Die Zeitschrift für Missionsarbeit, „Paulus" (ediert vom Lahn Verlag in Limburg), hat ebenso vor kurzem ihren Titel geändert; jetzt heißt sie „Signum“. Ist nicht auch das ein signum temporis?

Im französischen Sprachraum kam es zu keinen größeren Änderungen. „Revue NéoScolastique" - 1894 von Kardinal Mercier gegründet - nennt sich heute "La

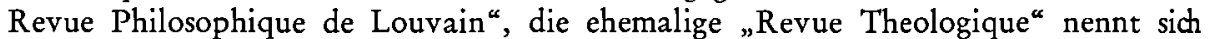
heute „Nouvelle Revue Théologique“. Die belgische Monatszeitschrift „Foyer Notre-

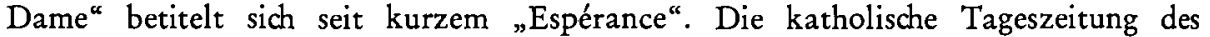
Bistums Lille „La Croix du Nord“ erscheint seit 1969 nur noch als Wochenzeitung mit dem Titel "La Croix-Dimanche“. Im selben Jahr taten sich die ehemaligen Zeitungen "Panorama Chrétien“ und „Chrétien Au-jourd'hui“ zusammen unter dem kombinierten Titel „Panorama Au-jourd'hui“. Das bekannte Pariser Verlagshaus „La Maison de la Bonne Presse" verzichtete auf den alten Namen zugunsten des neuen: „Bayard-Presse“.

1965 versuchten die französischen Ordensleute ihre missionarischen Anstrengungen in Einklang zu bringen, indem sie mit der Herausgabe verschiedener eigener Missionsblätter aufhörten und ein neues gemeinsames Blatt mit dem Titel „Peuples du Monde" gründeten. Die Zahl der Subskribenten war geringer als erwartet, und so hatte sich die neue Edition im Herbst 1971 zum Teil der ältesten Missions-Zeitschrift der Welt, den "Annales de la Propagation de la Foi“ anzuschließen, die bereits 1822 von Pauline Jaricot gegründet worden ist.

Ahnliche Kooperationsversuche der Missionszeitschriften unter neuem Titel sind auch in Deutschland zu verzeichnen ("Kontinente “), in Italien ("Populi e Missioni“), in Spanien ("Tercer Mundo") und in Holland ("Bijeen. Maandblad over internationale samenwerking, bijzonder op het terrein van missionering, zending en ontwikkelingswerk“, 1967 durch Fusion von „Katholieke Missiën“ [seit 1876] und einer Anzahl weiterer volkstümlicher Missionszeitschriften gegründet).

Aus dem englischen Sprachraum sind mir folgende Fälle bekannt: 1968 hörte das Blatt für englische Katholiken des Erzbistums Montreal in Kanada, „The Challenge" $\mathrm{zu}$ erscheinen auf, sein Nachfolger bekam den Titel "The Bridge". In Irland hat die Monatszeitschrift „Bulletin of the Catholic Communications Institute of Ireland“ 1970 den neuen, neutralen, kürzeren Titel, „Intercom" bekommen. Die älteste amerikanische katholische Zeitschrift, „Ave Maria“ (Notre Dame, Indiana) taufte sich $1970 \mathrm{zu}$ „A.D. 1970" um. Im selben Jahr hat sich die protestantische Zeitschrift "The Pulpit" zu "The Christian Ministry" unbenannt und stellte sich als Blatt für die Priester aller christlichen Gemeinschaften vor. 
Das Phänomen der Titeländerungen ist also für die katholische Presse nichts Spezifisches. Man kann es bei allen Konfessionen bemerken, besonders bei den Protestanten. Hier einige Beispiele aus ihrer Welt: Die bekannte evangelische Zeitung „Christ und Welt" nennt sich seit 1971 „Deutsche Zeitung“, sie bewahrte jedoch ihren alten Titel als Untertitel. Im selben Jahr schloß sich „Der evangelische Sonntagsbote“ dem Blatt "Weg und Wahrheit" an und verzichtete auf seinen alten Namen.

Die schweizerischen protestantischen Blätter "Schweizer Evangelist" und „Der evangelische Botschafter" vereinigten sich zu Beginn des Jahres 1968 unter dem neuen Titel "Kirche und Welt".

Und wie sieht es bei uns in Jugoslawien aus? In Kroatien gab es nicht viele Annderungen, weil uns dies die bescheidene Verlagstätigkeit gar nicht erlaubte. Das einzige Beispiel einer Änderung erlebte die Monatszeitschrift „Veritas"; sie behielt aber ihren ersten Titel im Untertitel mit der Ergänzung „Revue des heiligen Antonius" bei. Zu Beginn dieses Jahres hat „Vjesnik sv. N. Tavelic“" [Zeitschrift des heiligen Tavelić] (früher des „seligen") seinen Titel zu „Tavelić“ gekürzt und „Bakarska Svona" [Die Glocken von Bakar] heißen nun nur mehr „Die Glocken“.

Wenn man einige Zeitschriftentitel, wie sie vor dem Kriege lauteten, mit den heutigen vergleicht, kann man feststellen, daß folgende Zeitschriften ihre Titel geändert haben: "Obnovljeni Život" [Das erneuerte Leben] (früher "Život") [Leben]; "Maria " (früher "Der Bote der Frau von Sinj") und "Radosna Vijest" [Die Fröhliche Botschaft] (früher „Katholische Missionen").

Neben den Änderungen der Haupttitel ist die der Untertitel ebenso häufig zu verzeichnen. Wir haben schon auf die Metamorphose des früheren "Orbis Catholicus" hingewiesen. Etwas ähnliches ist auch bei der ökumenischen Zeitschrift "Catholica " passiert. Früher trug sie den Untertitel „Zeitschrift für Kontroverstheologie“, jetzt leißt sie „Zeitschrift für ökumenische Theologie“, was auch ihre Richtungsänderung andeutet.

Die französische, zweiwöchentliche Zeitung „Informations Catholiques internationales" begann unter dem Titel "L'Actualité religieuse dans le monde" zu erscheinen (1953-1955). Dann übernahm sie ihren heutigen Titel, trug aber den Untertitel "Bimensuel d'information religieuse". Vor zwei Jahren kehrte sie wieder zu ihrem ersten Titel zurück, und zwar setzte sie ihn als Untertitel.

In den Niederlanden haben fast alle katholischen Zeitschriften ihre Untertitel ge"ndert, was immer eine "Offnung zur Welt" andeutete. Die katholische Tageszeitung "De Volkskrant" [Volkszeitung] wechselte den Untertitel nicht, sondern ließ ihn einfach weg. Dieser wurde früher zu groß gedruckt; er hieß: „Katholiek Dagblad yoor Nederland".

Seltener geschah das Gegenteil, nämlich, daß als neuer kirchlicher Titel eine kirchlich engagierte Benennung gegeben wurde. Das haben in den USA zwei bischöfliche Blätter gemacht. Seit 1970 heißt "The West Virginia Register " "The Catholic Week", und "The North Central Louisiana Register" heißt nun "The Church Today".

In Deutschland gab es bis 1970 ein ausgesprochen konservatives Blatt: "Nunc et wemper"; aber im selben Jahr wurde daraus ein neues Blatt: „Der Fels“, dessen Redakteur Pater Gerhard Hermes war. Diesem Pater wurde die Redaktion seiner Drdenszeitschrift "Das Zeichen" untersagt, weil es hieß, daß er in seiner Tätigkeit tu reaktionär sei. 
Es ist mir nur ein einziger Fall bekannt - ich behaupte nicht, daß es nicht mehrere gäbe -, daß sich ein katholisches Blatt seinen alten - betont religiösen - Titel wieder hervorholte. Das geschah bei der kanadischen katholischen Wochenzeitung "The Catholic Register", die schon seit 150 Jahren erscheint. Eine gewisse Zeit hieß sie „The Canadian Register", kehrte aber 1972 zu ihrem alten Titel zurück.

\section{Omen}

Worauf kann man bei den oben angeführten Anderungen schließen? Welches sind eigentlich die Gründe, daß soviele religiöse Zeitschriften ihre alten Titel aufgeben und nach neuen suchen? Können wir auch dabei von der so häufig genannten „Identitätskrise" sprechen?

Nüchtern muß man vor allem zugeben: die Titeländerungen sind weder etwas Neues noch etwas Alarmierendes. Diese Erscheinungen gab es immer und wird es auch immer geben. Viele einzelne und Gemeinschaften finden in ihrer Vergangenheit oder in ihrem Programm nicht mehr genügend Inspiration und Rechtfertigung für ihre Arbeit. Die Welt um sie herum hat sich verändert und mit ihr auch sie selbst. Einige merken leider etwas später, daß sich nur die Umgebung um sie verändert hat, aber nicht sie selbst; jetzt machen sie große Sprünge, um mit der Zeit Schritt halten zu können. Die sozialen (R)evolutionen haben uns vor neue Aufgaben gestellt, und wir sind uns dessen bewußt, daß uns die alten Arbeitsmethoden nicht mehr helfen können. Auf der Suche nach der Lösung und Veränderung können wit manchmal nicht richtig das Ziel von den Mitteln und das Wesentliche vom Unwesentlichen unterscheiden.

Bei manchen Gläubigen, die durch die Krisen und Erneuerungen, die wir erleben, aufgeschreckt sind, ist die Kontinuität des Glaubens, der Bräuche und Traditionen unterbrochen worden. Journalisten und Schriftsteller unter den Katholiken sind besonders der Versuchung zum Sensationellen, zum Progressiven und zur Anpassung an die Welt um jeden Preis ausgeliefert. Bei einigen anderen ist die innere Identität ihres Ichs gebrochen. Sie sind nicht mehr das, was sie früher waren; etwas in ihnen ist gebrochen. Der Glaube, die Moral? Ihre Prinzipien, ihre Philosophie?

Die redlichen Wahrheitssucher versuchen ihre Praxis mit ihren Prinzipien in Einklang zu bringen (Orthodoxie), und deswegen brauchen sie neue Theorien, die sich in neuen Programmen ausdrücken. (Eben dies kommt oft in Neubenennungen der Zeitschriften zum Ausdruck.) Die Schwächeren sind jedoch geneigt, ihre Philosophie nach dem eigenen Leben oder nach soziologischen Gegebenheiten zu gestalten (Orthopraxis: das Milieu gewinnt die Oberhand); so brauchen auch sie neue Zeitschriften und neue Parolen.

Es sind aber nicht alle diese Anderungen auf dem Boden der philosophischen, ideologischen und religiösen Motive entstanden. Es gibt auch ganz prosaische Ursachen, z. B. Eingehen auf Modeerscheinungen. Man muß mit der "Weltpresse“ Schritt halten, zeitgenössisch und à la mode sein. Es sind nicht Prinzipien wichtig, sondern Anerkennung von Seiten der Kollegen und des Publikums. Man will die Leser durch immer neue Interessantheiten und neue Leser mit frischen Sensationen gewinnen. Deswegen auch muß der Titel verblüffend, ungewöhnlich und neu, wenn möglich nicht allzu klerikal oder religiös sein. 
Viele Titeländerungen werden aus rein praktischen Gründen vorgenommen, z. B. wegen schlechter finanzieller Lage, Schulden, zu kleiner Zahl der Abonnenten, um neue Leser zu gewinnen. Die wirtschaftliche Situation verlangt ein neues Außeres, neuen Inhalt und neue Titel. Einige Titel waren $\mathrm{zu}$ lang, und so mußte man sie kürzen (z. B. die früheren Titel von „De Heraut“ und „De Bazuin“), einige andere waren inhaltlich $\mathrm{zu}$ eng, und so mußte man sie erweitern ("Christlicher Sonntag" wurde zu "Christ in der Gegenwart", „Stimmen aus Maria Laach" wurde zu „Stimmen der Zeit", "Glasnik Gospe Sinjske" [Der Bote der Frau von Sinj] wurde zu "Maria“ und ähnliches). Es gab auch Zeitschriften, die ihren Titel verändern mußten - aus verschiedenen Gründen: z. B. „Veritas“ (das frühere Blatt wurde verboten, der Redakteur verurteilt) oder „Obnovljeni Život" [Erneuertes Leben] (denn es wurde wirklich erneuert, und obendrein existierte zur Zeit der Erneuerung in Sarajevo schon eine literarische Zeitschrift namens "Zivot ${ }^{*}$ ).

Die Programmerweiterung und das Stellen neuer Aufgaben waren Ursachen mancher Titeländerungen. Neben den schon erwähnten "Stimmen der Zeit" war das bei "Revue philosophique“ und bei "Theologie und Philosophie" der Frankfurter Jesuiten der Fall. Aus demselben Grund wurde auch aus "Paulus" „Signum“. Derselbe Gedanke leitete auch die Redaktion von "Tavelićc", als sie sich entschloß, den anderen Heiligen und Seligen des kroatischen Volkes mehr Aufmerksamkeit zu schenken. ${ }^{3}$ Okumenische Ursachen führten bei der "Catholica“ dazu, einen ganz neuen, dem alten völlig entgegengesetzten, Untertitel $\mathrm{zu}$ nehmen. Dasselbe galt auch für das Auslassen von (Unter) Titeln von „Orbis Catholicus“ und „Herder Korrespondenz“.

Die meisten Titeländerungen waren jedoch ideeller (um nicht zu sagen ideologischer) Natur. Die humanistische, oder genauer gesagt anthropozentrische Strömung unter den christlichen Intellektuellen veränderte manches in Redaktionen und manche Titel (so z. B. bei "Neues Hochland"). "Die Offnung zur Welt", das so oft unter Katholiken nach dem Zweiten Vatikanischen Konzil betonte Schlagwort, führte bei einigen Blättern zu Titel- und Inhaltsänderungen ("Speling “, „Heraut", „Werkhefte“ u. a.). Titeländerungen wurden aber nicht nur durch sog. progressive Tendenzen unter den Katholiken veranlaßt; es gab auch gegenteilige Fälle. Die konservativen Katholiken wollten mit den Titeländerungen ihre Treue $\mathrm{zu}$ den kirchlichen Traditionen hervorheben. So entstanden „Der Fels“, „Das Zeichen Mariens“ und noch einige andere.

Beim Aufzählen von Gründen für die Titeländerungen brachten wir der Illustration wegen einige Titel mit bestimmten Tendenzen in Zusammenhang; es versteht sich aber von selbst, daß für das Umbenennen einiger Zeitschriften mehr als ein Grund ausschlaggebend war. Es gab mehrere Gründe, und zwar ökonomische und ökumenische, aber auch programmatische und ideologische. In solchen Fällen kam es schneller und leichter zu Anderungen. Ich halte es jedoch für ganz sicher, daß trotzdem die Mehrheit dieser Veränderungen aus inner-religiösen und apostolischen Beweggründen erfolgte. Die Redakteure haben es ehrlich gemeint, und sie wollten ihrer Kirche nur Gutes. Natürlich hat nicht jeder dieser Versuche ganz gelingen können. Es kamen auch Fehler vor, die zu Konfusion und Desorientierung führten. Einige Katholiken sehen in Veränderungen nur Übel. Hier ein Beispiel, wie die Verweigerung der Änderung zum Ǔbel führen kann: Das Bistum Kansas-City in den

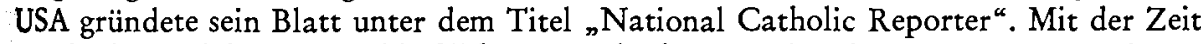
wurde die Redaktion ausschließlich Laien überlassen. Diese begannen zu opponieren, und ihre journalistische Arbeit orientierte sich ausschließlich "Progressiv“. Nachdem bischöfliche Mahnungen nichts erreicht hatten, verlangte der Bischof von der Redak- 
tion, den Titel des Blattes zu ändern, d. h. aus dem Titel das Wort „Catholic“ zu entfernen, weil dieses Blatt nicht mehr die reine katholische Lehre vertrete. Wie die Entwicklung weiterging, hat "Communicatio Socialis" berichtet." Manchmal kann auch der alte Titel täuschen. So haben die "Friedensgeistlichen" in der Tschechoslowakei ihre Wochenzeitung unter dem Titel "Katholische Zeitung" herausgegeben. In der Periode des Prager Frühlings haben die Bischöfe die Verwaltung des Blattes in ihre Hände genommen und nannten es "Christ und Welt", denn sie meinten, daß der alte katholische Name kompromittiert worden sei. Nach dem Einmarsch der sowjetischen Truppen begann es jedoch wieder als "Katholische Zeitung " zu erscheinen.

Ist daher das Nomen eines Blattes immer auch zugleich sein Omen? Wir haben gesehen, daß dies manchmal zutrifft, oft aber nicht. Wie die Menschen, so sind auch die Blätter nicht immer das, was uns ihr Name sagt. Mehr als nach dem Titel muß man sie nach ihrem Geist beurteilen: "Ihr werdet sie an ihren Früchten erkennen" (Mt. 7, 16, 20).

\section{Anmerkungen:}

1. Dieser Beitrag erschien zuerst in der jugoslawischen Vierteljahresschrift "Crkva u svijetu" [Kirche in der Welt], Split, Nr. 3/1973, unter dem Titel "Nomen - omen? (O pojavi mijenjanja imena nekih vjerskih listova) " [Nomen - omen? Über das Phänomen der Titeländerungen bei religiösen Blättern]. Die Übersetzung besorgte Alenka Kemptner (Salzburg).

2. Die meisten Notizen über Titeländerungen hat der Autor in "Communicatio Socialis" gefunden.

3. Anmerkung der Obersetzerin: Der eigentliche, hier verschwiegene Grund war das wachsende Nationalgefühl der Kroaten, das sich in diesem Fall auf einen einzelnen Heiligen, nämlich den heiligen Tavelić, konzentrierte.

4. Vgl. Klaus Hesper: Kirchenamtliche Distanzierung vom „National Catholic Reporter“, in: CS 2:1969, 143-145.

\section{S U M M A R Y}

As time went by some unrest has come into the Church. Beside new publications for the Church, also with the existing publications there are considerable changes, e.g. the church press has acquired a new outlook. This becomes especially clear in the change of titles of these publications. Beside changes of the main titles also the sub-titles are often changed. But the name (nomen) alone is not always an "omen". More important than the external view of the publication is the content and the spirit contained therein.

\section{RÉSUMÉ}

A une époque où tout se tranforme, l'agitation a aussi gagné l'Eglise. A côté des nouveaux moyens de communication de masse dont l'Eglise se sert, il se produit aussi des transformations visibles dans les anciens. La presse religieuse, elle-aussi par exemple, a reçu un nouveau visage. Cela est particulièrement visible, ne serait-ce que dans les changements des titres des publications religieuses. Outre les transformations des grands titres, on note aussi celles, tout aussi fréquentes, des sous-titres. Mais le nom d'une feuille n'est pas non plus toujours de bon augure. On doit juger les publications plus d'après le contenu et l'esprit que par l'apparence. 


\title{
RESUMEN
}

Con los cambios de los tiempos irrumpieron intranquilidades en la Iglesia. Se produjeron visibles renovaciones, no sólo en los nuevos medios de comunicación social de que se sirve la Iglesia, sino en los antiguos, como por ejemplo en la prensa de la Iglesia, que presenta hoy una nueva fachada. Ello se aprecia especialmente en los cambios introducidos en los titulares de encabezamiento, e incluso en los secundarios. Sin embargo el nombre de un rotativo no es siempre un augurio. Más que por su presentación hay que juzgar les publicaciones por sur contenido y espíritu.

\section{Erscheinungsformen der Kontroverspublizistik religiöser „Parteien` im Reformationszeitalter}

\author{
von Heinz-Dietrich Fischer
}

Während in der Antike die Rhetorik im Mittelpunkt aller Publizistik stand, wurde die mittelalterliche $z$ wischenmenschliche Kommunikation von der religiösen Aussage geprägt, die jedoch in einer ihrer Darbietungsformen - der Predigt - in gewisser Weise an die antike Rhetorik anknüpfte, - zumal das Lateinische weiterhin maßgebliche Sprache in Politik und Wissenschaft blieb. Abgesehen von Rede und Streitgespräch, von Botschaften und Heroldsaufrufen als Mitteln der politisch-publizistischen Verständigung erlitt die politische Publizistik im Mittelalter jedoch einen Rückschritt gegenüber den politisch-polemischen Streitformen der Antike. Gründete sich die Publizistik der Antike ,auf den Pluralismus der sozialen Gebilde und den Rationalismus des Denkens, so wuchs die mittelalterliche Aussage aus dem Geist eines umfassenden religiösen Gemeinschaftsbewußtseins, das die Mittel der Aussage mehr der Lehre des gesicherten Glaubens als der Mitteilung neuer Bewußtseinsinhalte widmete ${ }^{\prime 1}$ In diesem Rahmen konnte sich eine politische Publizistik nur in Ansätzen und sporadisch entwickeln. Erst zum Beginn der Neuzeit, nachdem Gutenbergs Erfindung eine neue Zeit eingeleitet und damit der Reformation durch das gedruckte Wort ein starkes Kampfmittel verschafft hatte, sind Ansätze zu neuer politischer Publizistik erkennbar.

Allein schon die Thesen Luthers von 1517, dessen publizistische Intention zunächst auf die Klärung theologischer Fragestellungen gerichtet war, dann aber schnell zu einem Politikum wurde und über den begrenzten sozialen Raum schnell in die breiteste Offentlichkeit gelangte, stellten letztlich einen „weiterwirkenden Katalysator für offfentliche Meinungsbildung ${ }^{\prime 2}$ schlechthin dar. Durch die drucktechnische Verbreitung der lutherischen Thesen und Schriften konnten sich von 1517 bis zum Reichstag zu Worms (1521) breite Kreise des Volkes der neuen Lehren annehmen.

Dr. Heinz-Dietrich Fischer ist Professor an der Sektion Publizistik und Kommunikation an der Ruhr-Universität Bochum. 
In der Agitation der beginnenden religiösen Auseinandersetzungen spielte somit neben der Rhetorik namentlich das gedruckte Wort eine wesentliche Rolle. Welch ein Förderungsmittel für die Reformation überhaupt die Buchdruckerkunst gewesen ist, kann heute leichter unterschätzt als überbewertet werden. Ein damals ziemlich weit verbreitetes Schlagwort stellte den Buchdruck sogar dem Druck der päpstlichen Herrschaft gegenüber. ${ }^{3}$ Erstaunlich schnell vollzog sich die Verbreitung der lutherischen Lehren. Auf der Frankfurter Messe von 1520 soll ein Buchhändler allein 1400 Exemplare von Luthers Schriften verkauft haben. ${ }^{4}$ Centgraf, der die Auflagenhöhe der lutherischen Schriften zwischen 1517 und 1522 überschlägig errechnet, gibt für nachgewiesene 500 Ausgaben bis Ende 1520 rund 500000 Exemplare an, die bis Ende 1522 sogar auf 800000 - ohne Postillen und Einzelpredigten - angestiegen sein sollen. ${ }^{5}$ Wenngleich diese Ziffern mit der üblichen Vorsicht zu betrachten sind, so war doch eine "vorher noch nie gewesene Publizität "в bei den lutherischen Reformationsschriften $\mathrm{zu}$ verzeichnen.

Angeregt von der sich vom Mittelalter abhebenden neuen Form der oppositionellen Meinungspublizistik Luthers kamen schon bald zur Zeit des Regensburger Reichstages (1541) und des Schmalkaldischen Krieges (1546/47) sogenannte ,Historische Volkslieder' auf, die Günter Kieslich in einer Studie in ihrer politisch-publizistischen Funktion für jene Zeit umrissen hat.

Während im historischen Lied des Mittelalters politische Begebenheiten, welche sich mit dem Kampf verschiedener Interessen und vieler Teilnehmer auseinandersetzen, kaum zu finden sind - die politischen Sprüche Walthers von der Vogelweide stellen eine nicht zu verallgemeinernde Ausnahme dar -, wurde im Reformationszeitalter das ,historische Lied zum politisch-publizistischen, zum beeinflussenden ... Lied ${ }^{\text {“7 }}$ politischer Strömungen. Die politische Publizistik jener Zeit wurde bestimmt von dem Kampf zwischen Katholiken und Protestanten. Zur Zeit des Regensburger Reichstages wurde publizistisch leidenschaftlich von beiden religiösen ,Parteien' um den Begriff der ,Nation' und alle damit zusammenhängenden Fragen gerungen. „Nation', so argumentierte die protestantische Partei, findet ihren Ausdruck nicht mehr in den Ordnungen des ,Heiligen Römischen Reiches Deutscher Nation', im Kaiser, in den Reichsfürsten, den Ständen und im kaiserlichen Kammergericht ..., gegen die Kritik erlaubt, ja geboten ist, weil sie dem neuen Begriffsinhalt und der neuen Vorstellung der ,Nation' nicht mehr entsprechen ... Im schroffen Gegensatz dazu stehen die Beweisführungen der katholischen Publizistik “, die erklärt, die Protestanten hätten es geradezu auf die Zerstörung des ,Heiligen Römischen Reiches abgesehen. Gleichsam als ,cantus firmus' "zieht sich der Aufruf zur nationalen Pflichterfüllung durch die Aussagen der Katholiken " ${ }^{\text {, }}$, resümiert Kieslich. Die dergestalt verbreitete Reimpublizistik sah sich schon bald den Verfolgungen ausgesetzt. Von Moritz von Sachsen ist bekannt, daß er im Sommer 1546, da er gerade im Begriff war, von der evangelischen zur Seite Karls V. überzuschwenken, ein Mandat an die Stadt Leipzig unter dem Datum des 9. Mai richtete, in dem er das Feilhalten deutscher Reime verbot, deren Urheber dem Kaiser „verdechtig und verdrießlich sein werdenn ${ }^{*} .{ }^{\circ}$

Nachdem der Augsburger Religionsfriede (1555) eine reichsrechtliche Gleichberechtigung der Protestanten neben den Katholiken erbracht hatte, war auf deutschem Boden zunächst eine gewisse Beruhigung in der polemischen Publizistik beider Seiten zu verzeichnen. Anders in Frankreich, wo seit 1562 die Hugenottenkriege den poli- 
tischen Niederschlag des auch dort geführten konfessionellen Streites bildeten. Den Höhepunkt der ,parteiischen' Publizistik dieser Auseinandersetzungen erbrachte die blutige Bartholomäusnacht vom 24. August 1572. In den Schriften, die die Geschichte der hugenottischen Bewegung und der Bartholomäusnacht behandeln, zeigt sich der eminent politische Zug des französischen Calvinismus, der von Anfang an sichtbar wurde. Diesem politischen Zug, der durch die Persönlichkeit Calvins maßgeblich hervorgerufen wurde, "dieser tiefverwurzelten Kraft der ,Sektec ist es zu verdanken, daß sie sich nicht durch das Blutbad in Frankreich ganz niederwerfen ließ, sondern im Gegenteil sofort wieder zum Angriff auf den verhaßten Katholizismus und das tief verachtete und verabscheute Königtum vorrückte. Dabei stellten die Schriftsteller der Partei das ganze Wissen ihrer Zeit in ihren Dienst, so daß diese Publizistik geradezu ein Dokument für die Bildung der Hugenotten ist"10, stellt Elkan fest. Erst das Edikt von Nantes (1598) bringt einen gewissen Abschluß des politischpublizistischen Kampfes der Hugenotten gegen Katholizismus und Königtum. In Deutschland schienen die religiösen Bewegungen seit 1555 eine allgemeine Beruhigung gefunden zu haben, es schien, als habe sich der neue evangelische Glaube die Anerkennung der alten Kirche erkämpft; aber diese Anerkennung war nur eine scheinbare. So war denn auch die Eintracht, welche um die Mitte des 16. Jahrhunderts zwischen den religiösen ,Parteien' ${ }^{\prime} \mathrm{zu}$ herrschen schien, nicht von anhaltender Dauer. Es war noch in der Erinnerung des Volkes, welche Wirkung Luther, namentlich in den ersten Jahren seines Auftretens, durch seine Broschüren und fliegenden Blätter auf die Massen des Volkes ausgeübt hatte. Bald warben aber auch die Katholiken mit ähnlichen Publikationen um die Gunst der Bevölkerung, wobei namentlich die Jesuiten zu Vorkämpfern der publizistischen Aktionen zur Rekatholisierung wurden. Diese publizistisch-politischen Kämpfe in den letzten Jahrzehnten vor Ausbruch des Dreißigjährigen Krieges veranlassen Krebs zu dem bedeutungsvollen Schluß: „Einen großen Teil der Schuld, daß die religiösen Gegensätze in Deutschland, trotz aller Friedensliebe und Kriegsfurcht des einzelnen, schließlich doch im Dreißigjährigen Krieg endeten, trägt die Publizistik “ beider "Parteien ' in den voraufgegangenen Jahrzehnten. „Durch fortwährendes Betonen dessen, was die Deutschen trennte, durch unablässiges Schüren der Zwietracht wurden die Gemüter allmählich so erhitzt, der gegenseitige Argwohn und Groll so gesteigert ${ }^{\alpha 11}$, daß schließlich die kriegerische Auseinandersetzung dieser politisch-publizistischen Kämpfe als einziger Ausweg vorhanden zu sein schien.

Als sich der Gegensatz zwischen Katholiken und Protestanten erneut in bedrohlicher Weise zuspitzte, schlossen sich die protestantischen Fürsten unter Führung des Kurfürsten Friedrich V. von der Pfalz im Jahre 1608 zur protestantischen Union zusammen, gegen welche die katholischen Reichsstände die Katholische Liga im Jahre 1609 gründeten. Dadurch waren relativ ungefestigte religiös-politische Lager entstanden, die sich ab 1618 infolge des Aufstandes der Stände zu Prag (,Prager Fenstersturz') kriegerisch und publizistisch aufs erbittertste bekämpften.

Jede der ,Parteien' behauptete von der Gegenseite, daß sie die höchsten Werte der Zivilisation mißachte und die übelsten Greueltaten begehe, wie Kindesmord, Folterung, Vergewaltigung, Raub und Brandstiftung; jede behauptete von sich, daß "Gott, alle guten Christen, ja alle guten Menschen auf ihrer Seite stünden ". ${ }^{12}$ Das erwachende Nationalgefühl wurde in Liedern angesprochen, die alles Deutsche als uberlegen, gut und edel bezeichneten. Besonders beliebt und für die Zeit charakteristisch war diejenige Form der Allegorie, welche die Ereignisse mit Hilfe von Wap- 


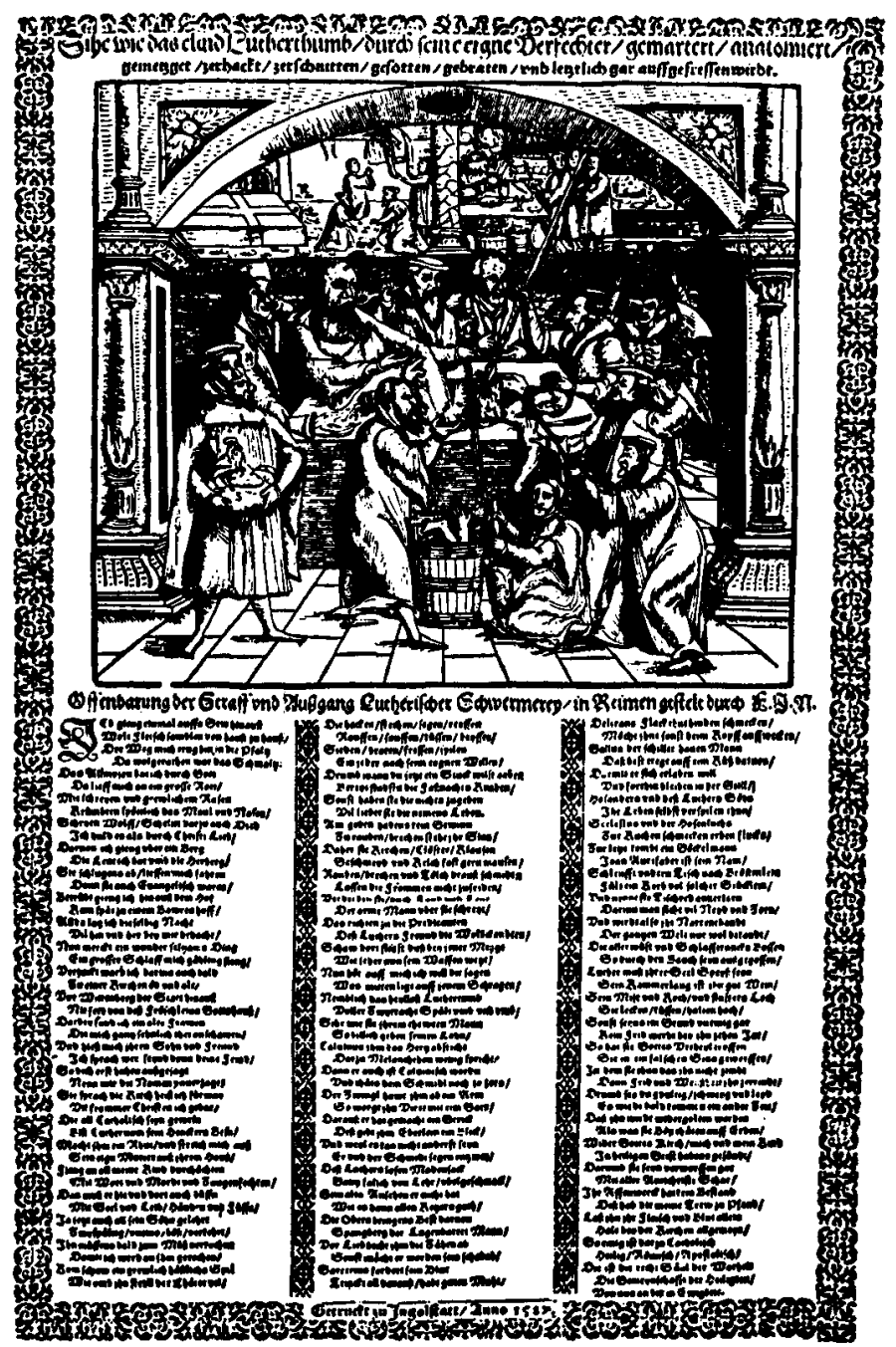

Katholisches Flugblatt aus dem Jabre 1587, erschienen in Ingolstadt, dem Zentrum des jesuitischen Propagandakampfes gegen die Reformation. Luther wird von politischen Gegnern seziert; - eine katholische Reaktion auf äbnlichgeartete protestantische Flugblätter, die mißbandelte Mönche in äbnlichen Situationen zeigten. 


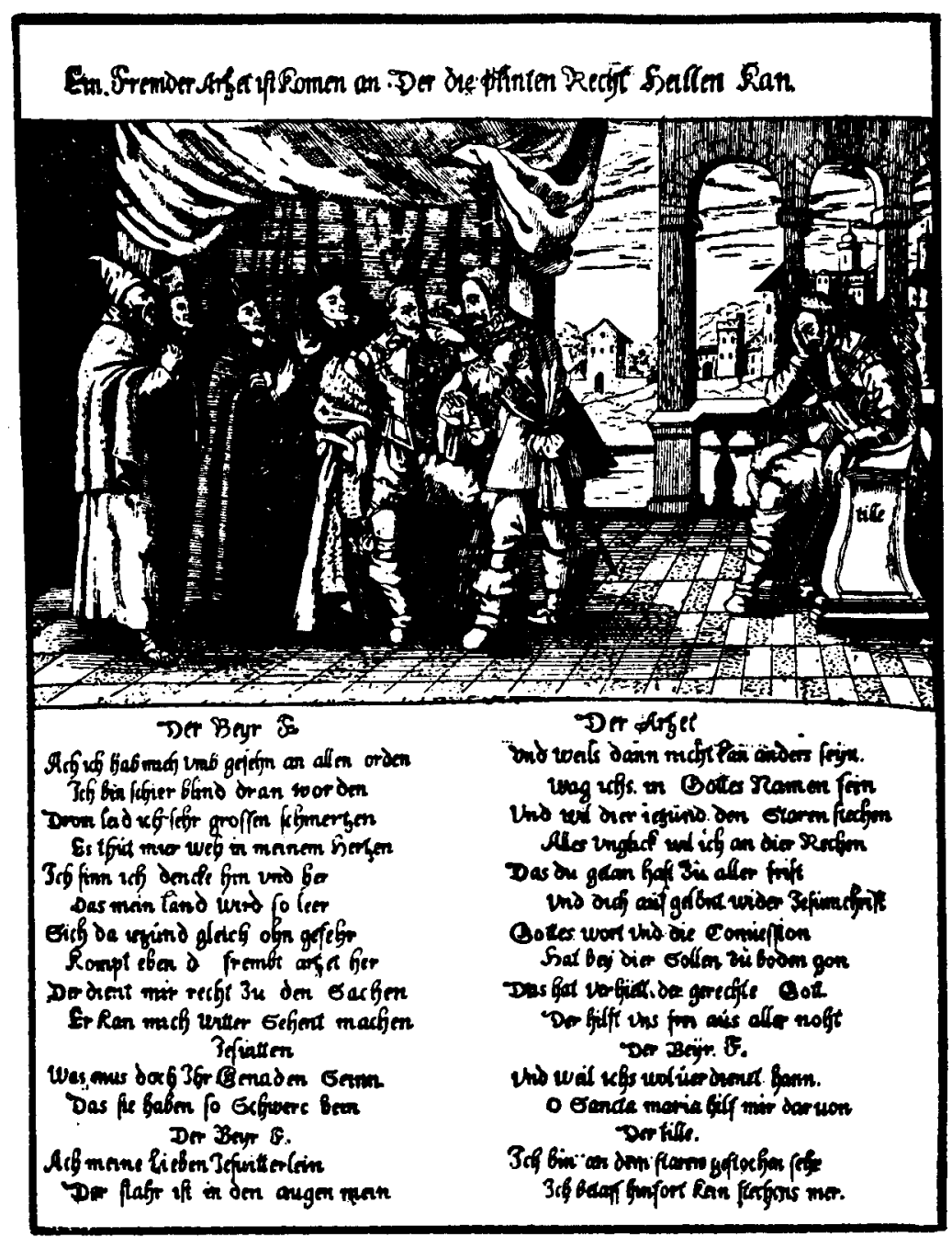

Protestantisches Flugblatt des Winters 1631/32 feiert Gustav Adolf als den fremden Arzt, der die katholischen Länder zu beilen imstande sei. Nach dem Siege Gustav Adolfs (Artzet) Wher Tilly (Tille) richtet sich dieser gegen den von Jesuiten beeinflußten Maximilian von Bayern (Beyr), den bayerischen Kurfürsten. 
pentieren oder sonstigen symbolischen Tiergestalten darstellte. Der Adler bedeutete stets den Kaiser, der Bär Maximilian von Bayern, die holländischen Generalstaaten wurden als Zentauren dargestellt, Mansfeld als Leopard, die Ungarn als Hasen, die Böhmen als Schafe, der kaiserliche General Spinola als Spinne etc.

Charakteristisch für das politische Lied in der Zeit des Dreißigjährigen Krieges ist die Form der Parodie: die Lieder begannen häufig mit den Anfangsworten geistlicher und weltlicher Lieder, oder es wurden geistliche Lieder, Gebete und Bibelstellen der Form und auch dem Inhalte nach für die politische Aussage umgestaltet, wodurch zweifelsohne eine rasche Erlernbarkeit und schnelle Verbreitung gesichert waren. ${ }^{13}$

Kurz vor Ausbruch des Krieges sind in Deutschland auch die ersten Periodika zu verzeichnen, so vom Jahre $1609 \mathrm{ab}$ die Straßburger, Relation aller fürnemmen und gedenkwürdigen Historien usw. ${ }^{14}$ sowie der Wolfenbütteler ,Aviso ${ }^{15}$. In den folgenden Jahren erschienen zusätzlich mehrere Blätter u. a. in Frankfurt, Leipzig, Berlin, Wien, München und Köln. Salomon bemerkt, daß bei diesen frühen Zeitungen "trotz ... trocken referierenden Tones ... ein aufmerksames Ohr dennoch aus den verschiedenen Zeitungen heraushören “ könne, „aus welchem Lager sie hervorgegangen sind, welcher Partei sie im Grunde angehörten ... Die Zeitungen von Straßburg, Augsburg, Frankfurt a. M., Leipzig und Berlin tragen entschieden einen protestantischen Charakter ... Die Zeitungen von Wien, München und Köln haben eine katholische Grundstimmung "18. Gegen diese These, daß es sich hierbei gewissermaßen um frühe Erscheinungsformen der Parteipresse gehandelt haben könnte, wendet sich entschieden Bauer, wenn er am Beispiel der Straßburger ,Relation ...' ausführt: „Schon von jener $1609 \mathrm{zu}$ Straßburg erschienenen ,Relation aller fürnemmen und gedenkwürdigen Historien usw." " wird behauptet, "daß ihre Berichte einen deutlich protestantischen Charakter verrieten, ohne auf die inneren Streitigkeiten der verschiedenen Theologen Acht zu geben. Damit ist nun freilich die Tatsache der Tendenz gegeben, aber nicht die der Parteipublizistik. Die Zeitung empfing aus protestantisch gesinnten Kreisen ihre Nachrichten und war also in höherem Grade der empfangende als der gebende Teil. Ein Parteiblatt muß aber aktiv die Lehren einer bestimmten Gruppe gleichgesinnter Menschen vertreten, muß deren Anschauungen auch anderen mundgerecht zu machen suchen, muß kämpfen und werben "17, aber das traf für die Straßburger ,Relation' in keiner Weise zu. So findet sich in den Frühformen der Presse zwar im Ansatz eine Tendenz, doch kann sie keinesfalls als ,die Parteimeinung der Katholiken oder Protestanten jeweils aufgefaßt werden, da beide Konfessionen nicht als Parteien im modernen Sinne aufzufassen sind.

Wohl um einiges meinungsfreudiger und ,parteiischer' für die eine oder andere Seite waren jedoch die zahlreichen Flugschriften während des Dreißigjährigen Krieges, die eine unverhältnismäßig stärkere Resonanz fanden als die Wochenblätter. ${ }^{18}$ Schon der böhmische Aufstand von 1618 entfachte eine reichhaltige Flugschriftenpublizistik, die mit der ,Apologia oder Entschuldigungsschrift ${ }^{\circ}$ der evangelischen Seite in bezug auf den Fenstersturz der beiden kaiserlichen Statthalter eingeleitet wurde. ${ }^{19}$

Infolge Repressalien gegen die Autoren von Flugschriften ging man bald mehr und mehr dazu über, solche Schriften anonym erscheinen zu lassen. Auch griffen bald außerdeutsche Publizisten in den deutschen Konfessionskampf ein, so namentlich von schwedischer Seite. Mit der wachsenden Radikalisierung kam jedoch auch bald die politische Lüge in die evangelische Propaganda ${ }^{20}$, die ab 1621 in der Anhaltischen Kanzlei ihr Propagandazentrum hatte, denn "alle Fäden der antihabsburgischen und 
antikatholischen Politik ... liefen zusammen in der Kanzlei des Fürsten von Anhalt" ${ }^{\text {a21. }}$.

Ab 1622 trat für die katholische Liga die Spanische Kanzlei in ihren antiprotestantischen publizistischen Kampf ein. ${ }^{22}$ Beide Konfessionen schufen zahlreiche Schlagworte, um in etwa an die nationalen Emotionen der Bevölkerung zu appellieren, was insbesondere nach der Schlacht bei Nördlingen deutlich wurde. Die katholische Propaganda bezeichnete die Schweden als Eindringlinge, Rebellen und Friedensstörer, als des Heiligen Römischen Reiches und des deutschen Volkes Feinde, während in der Gegenpropaganda die protestantischen Armeen bei Nördlingen als Verteidiger der religiösen Freiheit gegen die Papisten auftraten. ${ }^{23}$

Unzählige Flugschriften, Berichte, Briefe, Proklamationen und sonstige Dokumente geben ein Bild, wie Volk und Stände während des Dreißigjährigen Krieges propagandistisch bearbeitet wurden. Wie umfassend die Flugschriftenliteratur für die Betrachtung jener Epoche ist, bemerkt Schottenloher: „Man könnte in der Tat mit der großen Fülle solcher Blätter die ganze Geschichte jener Zeit zum Ausdruck bringen. ${ }^{{ }^{24}}$

Gegen Ende des Krieges, als die allgemeine Resignation eine Publikationsmüdigkeit nach sich zog, waren insbesondere jene nunmehr zahlenmäßig geringeren Flugschriften von Bedeutung, die den Frieden verlangten, - die politischen und religiösen Gegensätze waren durch die allgemeine Not verdrängt worden..$^{25}$

\section{Anmerkungen:}

1. Walter Hagemann: „Grundzüge der Publizistik“, a.a.O., S. 123.

2. Siegfried von Kortzfleisch: „Die publizistische Bedeutung von Luthers Thesenanschlag “, in: ,Publizistik', Bremen, 5. Jg. (1960), Heft 3, S. 131/32.

3. Arwed Richter: „Uber einige seltenere Reformations-Flugschriften aus den Jahren 1523 bis 1525 ", Beilage zum Bericht über das Schuljahr 1898 bis 1899 der , Realschule auf der Uhlenhorst', Hamburg 1899, S. 44, wo ergänzend berichtet wird: „Die anonyme Flugschrift ,Ein clag und bitt der deutschen nation an den almechtigen got und erlosung aus dem gefenkniß des Antichrist' prägt den Gedanken in die folgende Form:

,Den druck uns Deutschen got zugeschickt hat

$\mathrm{Zu}$ lernen die schrift und erkennen der Romer art

Welch die heilige schrift wollen underdrucken"."

4. Karl Fischer: „Deutschlands öffentliche Meinung im Reformationszeitalter und in der Gegenwart“, Berlin 1874, S. 26, wo der Autor hinzufügt: „Nicht geringer war der Absatz von Melanchthons und Huttens Schriften ..., eine ganz besondere Tätigkeit entwickelte Hutten mit seiner eigenen Druckerei auf der Ebernburg. Luthers und anderer Schriften wurden gewöhnlich in kleineren Versammlungen vorgelesen und durchgesprochen: in Privathäusern, auf Plätzen und Märkten, in Trinkhäusern und Bädern. Anfang der zwanziger Jahre war es soweit gekommen, daß die Anhänger des Alten keine Verleger für ihre Sduriften mehr fanden ... Schreibt ja 1523 Erasmus an den König von England von Basel aus. ,Hier ist kein einziger Buchhändler, der es wagt, nur ein Wörtchen gegen Luther drucken zu lassen: aber gegen den Papst darf man schreiben, was man will:"“

5. Alexander Centgraf: "Martin Luther als Publizist" (Geist und Form seiner Volksführung), Bd. 14, Reihe A ,Zeitung und Zeit', N.F., Frankfurt/Main 1940, S. 19 f.

6. Siegfried von Kortzfleisch: "Die publizistische Bedeutung von Luthers Thesenanschlag", a.a.O., S. 134.

7. Günter Kieslich: „Das ,Historische Volkslied` als publizistische Erscheinung (Bd. 1, Studien zur Publizistik), Münster 1958, S. 117.

8. Daselbst, S. 107 f.

9. Wilhelm Bauer: „Die öffentliche Meinung in der Weltgeschichte“, a.a.O., S. $189 \mathrm{f}$.

10. Albert Elkan: „Die erste Publizistik der Bartholomäusnacht", phil. Diss. Heidelberg o.J., Heidelberg 1905, S. 21. 
11. Richard Krebs: „Die politische Publizistik der Jesuiten und ihrer Gegner in den letzten Jahrzehnten vor Ausbruch des Dreißigjährigen Krieges“, phil. Diss. Halle-Wittenberg 1890, S. 18.

12. Alfred Sturminger: „3000 Jahre politische Propaganda“, a.a.O., S. 163.

13. Daselbst, S. $163 \mathrm{ff}$.

14. Vgl. Walter Schöne (Hrsg.): „Die Relation des Jahres 1609“, Leipzig 1940.

15. Vgl. Wilhelm Hartmann: „Wolfenbüttel als Druckort des ,Aviso“ von 1609, der ältesten periodisch gedruckten Zeitung", in: ,Niedersächsisches Jahrbuch für Landesgeschichte, Bd. 31, Hildesheim 1959, S. 175 ff.

16. Ludwig Salomon: „Geschichte des deutschen Zeitungswesens von den ersten Anfängen bis zur Wiederaufrichtung des Deutschen Reiches", Bd. 1, Oldenburg-Leipzig 1900. S. $48 / 49$.

17. Wilhelm Bauer: „Die öffentliche Meinung und ihre geschichtlichen Grundlagen“, Tübingen 1914, S. 277.

18. Wilhelm Bauer: „Die öffentliche Meinung in der Weltgeschichte", a.a.O., S. 188.

19. Johannes Gebauer: „Die Publizistik über den böhmischen Aufstand von 1618“, phil. Diss. Halle-Wittenberg, Halle 1892, S. 3.

20. Diethelm Böttcher: "Propaganda und öffentliche Meinung im protestantischen Deutschland 1628-1636", in: ,Archiv für Reformationsgeschichte" (Gütersloh), 44. Jg., Heft 1/2 (1953), S. 202.

21. Reinhold Koser: „Der Kanzleienstreit“ (Ein Beitrag zur Quellenkunde des Dreißigjährigen Krieges), Halle 1874, S. 3.

22. Daselbst, S. $23 \mathrm{ff}$.

23. Göran Rystad: „Kriegsnachrichten und Propaganda während des Dreißigjährigen Krieges“, Lund 1960, S. $198 \mathrm{ff}$.

24. Karl Schottenloher: „Flugblatt und Zeitung“ (Ein Wegweiser durch das gedruckte Tagesschrifttum), Berlin 1922, S. 262.

26. Wilhelm Tekotte: „Beiträge zur Publizistik des Westfälischen Friedens“, phil. Diss. Münster 1934, S. 4 ff.

\section{S U M M A R Y}

At the beginning of modern time, a new period has begun with the invention of the printing press by Gutenberg. Thus with the printed word giving a strong means of fighting the reformation, a new political journalism begun. The reformation involved a strong journalism. Soon, especially Jesuits, became pioneers for journalistic actions for re-catholisation. Up to the "thirty-year War" the political journalistic battles became stronger. Only at the end of this war misery superseded the political-religious oppositions.

\section{RESUME}

C'est l'invention de l'imprimerie par Gutenberg, au début des temps modernes, qui introduisit une nouvelle époque et donna à la Réforme, par l'intermédiaire de la parole écrite, une arme puissante. Une nouvelle publicistique s'installa. La Réforme obtint une forte publicité. Ce sont particulièrement les Jésuites qui devinrent bientôt les champions en actions publicistiques en vue de la recatholicisation. Les combats polito-publicistiques s'échauffèrent jusqu'à la guerre de 30 ans. Ce ne fut qu'à sa fin que la misère supplanta les conflits politiques et religieux. 